\title{
Musculoskeletal Disorders and Menopause
}

\author{
Suvarna Satish Khadilkar ${ }^{1}$
}

Received: 26 February 2019 / Accepted: 26 February 2019 / Published online: 7 March 2019

(c) Federation of Obstetric \& Gynecological Societies of India 2019

\begin{abstract}
Menopause has an adverse impact on overall musculoskeletal health. It is associated with osteoporosis, osteoarthritis and Sarcopenia. Sarcopenia includes age-related muscle wasting as well as loss of muscle function. It is a relatively newly recognized condition and is known to be accelerated by estrogen deficiency. Osteoarthritis is also linked with estrogen deficiency more recently. Locomotor disability leads to a compromised quality of life. Sarcopenia and obesity (sarcobesity) have adverse outcome as it leads to morbidity due to increased incidence of lifestyle diseases like diabetes mellitus, hypertension. Poor musculoskeletal health may progress to frailty and higher incidence of falls and fractures which further increase associated morbidity and mortality. The healthcare providers and policy makers need to focus on this group of disorders and include its prevention in national program to reduce the health resources utilization.
\end{abstract}

Keywords Sarcopenia $\cdot$ Musculoskeletal disorders $\cdot$ Osteoporosis $\cdot$ Osteopenia $\cdot$ Sarcobesity $\cdot$ Frailty $\cdot$ Frail $\cdot$ Sarc-F . Osteoarthritis

\section{Introduction}

Musculoskeletal health of women is increasingly becoming a health hazard at midlife and beyond. Locomotor disability and frailty-falls-fractures lead to not only a great compromise of quality of life but substantially increase associated mortality in postmenopausal women. Osteoporosis is a wellrecognized, most prevalent bone disorder in postmenopausal women, characterized by low bone mineral density and deterioration of micro-architecture of bone. This risk is further potentiated by weak muscles, weak joints and frailty which together increase the risk of falls. Osteoarthritis is also a

Suvarna Satish Khadilkar MD DGO FICOG, CIMP, Diploma in Endocrinology (UK) is Editor in Chief of Journal of Obstetrics and Gynecology of India, and Treasurer, FOGSI; she is Consultant Gyne-Endocrinologist, and Professor and head of the Department of Ob-Gyn, Bombay Hospital and Medical Research Centre, Mumbai; Former Professor and Head, Department of Ob-Gyn, RCSM, Government Medical College, Maharashtra and Associate Professor and Unit Chief Grant Medical College and Cama and Albless Hospital, Mumbai.

Suvarna Satish Khadilkar

suvarnakhadilkar@yahoo.com;

suvarnakhadilkar2@gmail.com

1 Bombay Hospital and Medical Research Centre, 12

NewMarine Line, Mumbai 400020, India well-established, easy to diagnose condition which involves loss of cartilage. Sarcopenia is, however, a newly recognized condition which indicates age-related loss of skeletal muscle mass as well as function. Sarcopenia may further progress to frailty and sarcopenic obesity, which put these women under risk of compromised life as well as increased mortality. This editorial will take a quick review of various disorders of musculoskeletal system in women.

\section{Risk Factors Associated with Musculoskeletal Health}

There are many modifiable risk factors like alcohol intake, smoking, low body mass index, low calcium intake, vitamin D deficiency, insufficient physical activity, frequent falls, ergonomical, psychosocial and occupational factors, whereas non-modifiable risk factors include female gender, increasing age, family history, previous fracture, race or ethnicity, onset of menopause and prior hysterectomy. Women beyond 5 years of menopause, with late menarche and those having early menopause, are all at higher risk of musculoskeletal disorders. 


\section{Effects of Hormones on Musculoskeletal Health}

Sex steroids have a positive effect on musculoskeletal health of a men and women. Estrogen is important in a female body skeleton and connective tissues in general. Estrogen deficiency around menopause is bound to cause adverse effects on health of bones, muscles, ligaments tendons collagen, cartilage, synovial membrane and capsule of joints.

Apart from Estrogen, follicle-stimulating hormone, dehydroepiandrosterone (DHEA), growth hormone $(\mathrm{GH})$ and insulin and insulin-like growth factor-1 (IGF 1) stimulate the muscle protein synthesis and improve the activity of motor neurons. Estrogen acts directly and indirectly on muscle and contributes to pathogenesis of sarcopenia. Bioavailable testosterone is decreased in menopausal women. DHEA may contribute to the increase in muscle mass, the improvement in glucose and insulin levels and the decrease in fat mass and reduce the risk of breast cancer [1]. Other factors which contribute to the development of sarcopenia are increased inflammatory activity, oxidative stress, mitochondrial dysfunction, increased apoptosis, reduced physical activity and impaired nutrition.

Poor musculoskeletal health has multifactorial etiology, but hormones play a major role.

\section{Osteoporosis}

Osteoporosis is widely prevalent in India. Fifty million cases of osteoporosis were reported in 2013. Prevalence of vertebral fractures and hip fractures increases multifold with age in females [2]. WHO [3] defines normal bone mineral density (BMD) as within one standard deviation of mean bone density of young adult woman. Osteoporosis still remains underdiagnosed and undertreated in spite of availability of ample number of screening tests. Dualenergy X-ray absorptiomentry (DEXA) is the gold standard for assessment of BMD and diagnosis of osteoporosis. DEXA is recommended in women age 65 and older men above age 70 years, women and men over 50 years with risk factors or with a fracture [4]. Frax score (Fracture Risk Assessment Tool) is a useful tool which helps to calculate patient's 10 -year probability of fracture. It requires name of the country, BMD, age, gender and the clinical risk factors mentioned above. When the risk score of $\geq 3 \%$ for hip fracture or a score $\geq 20 \%$ for major osteoporotic fracture is observed, clinician should intervene [4].

Osteoporosis is largely a preventable and treatable disease, and postmenopausal osteoporosis can be prevented and treated with menopausal hormone therapy (MHT). But, after womens' health initiative (WHI) reports [5] universal MHT is no more recommended. Only the symptomatic women, women at high risk for osteoporosis need MHT, and will be benefitted for osteoporosis [6, 7]. Older women can have other options for intervention like raloxifene and bisphosphonates. Newer molecules like tissue selective estrogen complex can also be an option.

The WHI study ( $N=93,000$ postmenopausal women) analysis showed that the risk of fracture in women with type 2 diabetes had a $20 \%$ higher incidence than in women without type 2 diabetes in spite of having normal BMD scores in the beginning [8].

\section{Sarcopenia}

Sarcopenia is a relatively new terminology, evolved from Greek words "sarko" meaning skeletal muscle and "penia" meaning deficiency. In 1989, Rosenberg used the term "sarcopenia" to describe the age-related loss of skeletal muscle mass $[9,10]$. Sarcopenia is the loss of muscle mass and function associated with aging, whereas cachexia is the term used for weight loss due to an underlying illness. Both should be clearly differentiated as both are muscle wasting disorders in the aging population, but they go generally unrecognized. Another term "dynapenia" is used for loss of muscle strength.

After 50 years of age, approximate muscle loss can be expected to be $1-2 \%$ per year [11]. The prevalence of sarcopenia in women increases around the age of 50, whereas in men the prevalence increases at the sixth decade [12]. A cross-sectional study reported a decline in muscle mass of $0.6 \%$ per year after menopause [13]. Postmenopausal women have large amount of non-contractile muscle tissue, such as intramuscular fat, compared to younger women [14].

Consensus definition of sarcopenia given by European Working Group on Sarcopenia for Older People (EWGSOP) of appendicular skeletal muscle mass index (ASMI) of $<5.5 \mathrm{~kg} / \mathrm{m}^{2}$ is accepted [15]. Sarcopenia is characterized by atrophy of fast muscle fibers, type II fibers, by a decreased number of motor units and by accumulation of fat within muscle. In addition to above-mentioned risk factors, insulin resistance and insulin-like growth factor I are also implicated in pathophysiology. Muscle mass needs positive protein balance and pathways for synthesis (mTOR) and degradation (myostatin) of protein will lead to resultant muscle building or wasting, respectively.

EWGSOP recommended staging as "presarcopenia," "sarcopenia" and "severe sarcopenia." The "presarcopenia" stage is characterized by low muscle mass without influence on muscle strength or physical performance. This stage can only be detected by methods that measure muscle mass 
accurately. The "sarcopenia" stage is characterized by low muscle mass and low muscle strength or low physical performance. "Severe sarcopenia" is characterized by all three criteria of the definition (low muscle mass, low muscle strength and low physical performance). Classifying stages of sarcopenia is useful for planning management.

Typically patients complain of difficulty in rising from chair, climbing up and downstairs and in regaining lost balance. It is difficult for them to walk on uneven surfaces. Screening is recommended in patients with prolonged inactivity, weight loss, recurrent falls, comorbidities, especially diabetes mellitus, slow gait speed $<.8 \mathrm{~m} / \mathrm{s}$ over 4 min or any specific symptom suggestive of sarcopenia. Many diagnostic tools are available. Skeletal muscle mass index $=$ appendicular skeletal muscle mass/height ${ }^{2}$ (2 SD below mean of young adults) - men: $7.26 \mathrm{~kg} / \mathrm{m}^{2}$; women: $5.5 \mathrm{~kg} / \mathrm{m}^{2}$ by dual-energy $\mathrm{X}$-ray absorptiometry is one diagnostic parameter. Other tool like bio-impedance analysis may also be useful. SARC-F is a simple questionnaire developed for rapid diagnosis of sarcopenia [16] similar to FRAX score for osteoporosis.

Nutritional intervention like adequate protein and energy intake, nutrients supplements especially vitamin D supplementation is recommended. Aerobic exercises and resistance exercises are also recommended. Aerobic exercise improves muscle protein synthesis and muscle quality. It also reduces intramuscular fat. Resistance exercise training improves muscle mass and strength and attenuates development of sarcopenia. A Cochrane review of 121 randomized controlled trials suggests that progressive resistance therapy 2-3 times per week is the most beneficial exercise [17].

Estrogen supplementation should not be recommended as a primary line of treatment for sarcopenia in view of higher incidence of cardiovascular disease and breast cancer associated with the use of MHT [18]. However, if MHT is used in the window period in symptomatic women, it may help maintain bone and muscle health [6].

\section{Osteoarthritis}

Osteoarthritis is a slow-progressing joint inflammation that can result from cartilage degeneration. It is now recognized that cartilage degeneration can occur due to estrogen deficiency around the time of menopause. Increasing age, obesity, poor joint alignment, trauma and family history are among other risk factors. It can remain silent for years, before it becomes symptomatic. Pain, swelling, crepitus and stiffness of joints are common symptoms. Diagnosis is generally clinico-radiological. Plain radiograph is useful in making diagnosis. Degeneration and the presence of osteophytes aid the diagnosis.

It can influence quality of life because of locomotor disability and is a common cause of recurrent falls in midlife and elderly women. Lifestyle changes, weight reduction, dietary changes, exercises and supporting devices are primary measures to reduce the further progression of the disease and NSAIDs form the basis for symptomatic treatment to reduce inflammation and pain. There is enough data supporting benefit of MHT on progression of osteoarthritis [19]; however, some reports also state that MHT may in fact cause degenerative changes in cartilage [20]. Hence, with these conflicting reports, MHT should not form a first-line treatment against progression of osteoarthritis. However, MHT prescribed for other symptoms of menopause may be beneficial to women suffering from osteoarthritis.

\section{Sarco-Osteoporosis: One Disease Rather than Two?}

Common pathophysiological mechanisms exist between sarcopenia and osteoporosis. Mechanical and biochemical (endocrine and paracrine) communication through substances secreted by muscles (myokines) and by bones (osteokines) is suggested. There is good evidence for "an interconnected biology of bone and muscle with constant cross talking within a bone-muscle unit" [21]. It is possible that stages of presarcopenia, sarcopenia and severe sarcopenia may correspond with osteopenia, osteoporosis and severe osteoporosis, respectively.

\section{Sarcobesity}

Diminished muscle mass along with increased fat mass is called "sarcobesity." Sarcobesity in conjunction with dynapenia is manifested by impaired mobility, development of lifestyle-related diseases, type 2 diabetes, cardiovascular disease and metabolic syndrome [22, 23]. Inappropriate dietary advice for obese individuals can lead to deterioration of muscle mass and relative increase in the fat. Sarcopenia is commonly exacerbated in overweight and obese individuals. Diets that result in energy restriction in isolation lead to a reduction in both fat and muscle mass. Therefore, ultimately predisposes one to an unfavorable body composition. Sarcopenia itself leads to lack of exercise resulting into excessive weight gain. Aerobic exercise helps by bringing about beneficial changes in whole-body metabolism and reducing fat mass, while resistance exercise maintains lean (muscle) mass. Current evidence strongly supports the inclusion of resistance and aerobic exercise to complement mild energyrestricted high-protein diets for healthy weight loss as a primary intervention for sarcobesity [24]. 


\section{Frailty}

Fatigue, resistance, ambulation, illness and loss of weight are the factors contributing to frailty. Frailty is defined as a pre-disability condition where any three of the following five criteria are present $[25,26]$ which are as follows: unintentional weight loss, physical exhaustion, weakness (poor grip strength), slow motor performance (walking speed) and low physical activity. FRAIL, a simple five-item questionnaire $[27,28]$ for frailty, correlates well with SARC-F.

It is estimated that among older hospitalized patients, $28.1 \%$ females are frail [29].

\section{What Can be Done to Reduce the Burden?}

Focussing on preventive care is most effective way to reduce the burden. Healthy nutrition strategies, encouraging protein rich food and balanced diet, high calcium containing food items, nutritional supplements including vitamin D supplements, practicing regular aerobic and resistance exercise and promoting healthy lifestyle, are measures to be undertaken. Professional bodies should undertake awareness drive and community service programs.

A study done on awareness on musculoskeletal diseases throws more light on how midlife population is made aware about these disorders. Study reported that postmenopausal Indian women get their knowledge from the media (TV/ Radio): $55 \%$, friends/family: $20 \%$, newspapers: $20 \%$ and only $20 \%$ patients get information from the doctors [28]. There is a clear need to increase doctor participation for educating the patients so that they can avail of preventing health checkups.

Lack of awareness about the musculoskeletal disorders and their serious consequences is a major hurdle in implementation of strategies. If appropriate management strategies are not implemented timely, then these disorders will sequentially progress to "Disability-Disease-Death!"

\section{Conclusions}

Musculoskeletal diseases are prevalent in postmenopausal and aging women which compromise quality of life significantly and limit productivity. Estrogen deficiency around menopause is an important risk factor that accelerates development of both osteoporosis and sarcopenia. Bone and muscle loss share the same etiological factors; hence, preventive care should begin at menopause.MHT is still not considered for universal prevention but could benefit highrisk women and women having menopausal symptoms. We have National Programme for Health Care of the Elderly (NPHCE) in place, but we need to have robust national health program for prevention of osteoporosis and musculoskeletal disorders to reduce high health resource utilization. Increasing awareness among women at midlife and beyond more among the healthcare providers is extremely important and will go a long way to build the solid foundation for future India.

\section{References}

1. Labrie F, Luu-The V, Belanger A, et al. Is dehydroepiandrosterone a hormone? J Endocrinol. 2005;187(2):169-96.

2. Mithal A, Bansal B, Kyer CS, et al. The Asia-pacific regional audit-epidemiology, costs, and burden of osteoporosis in India 2013: a report of international osteoporosis foundation. Indian J Endocrinol Metab. 2014;18(4):449.

3. World Health Organization. World Health Organization scientific group on the assessment of osteoporosis at primary health care level; 2004.

4. Cosman F, de Beur SJ, LeBoff MS, et al. Clinician's guide to prevention and treatment of osteoporosis. Osteoporos Int. 2014;25(10):2359-81.

5. Writing Group for the Women's Health Initiative Investigators. Risks and benefits of estrogen plus progestin in healthy postmenopausal women: principal results from the Women's Health Initiative randomized controlled trial. JAMA. 2002;288(3):321-33.

6. Khadilkar SS. Post-reproductive health: window of opportunity for preventing comorbidities. J Obstet Gynecol India. 2019;69:1. https://doi.org/10.1007/s13224-019-01202-w.

7. Crandall CJ, Aragaki A, Cauley JA, et al. Associations of menopausal vasomotor symptoms with fracture incidence. J Clin Endocrinol Metab. 2015;100:524-34.

8. Bonds DE, Larson JC, Schwartz AV, et al. Risk of fracture in women with type 2 diabetes: the Women's Health Initiative Observational Study. J Clin Endocrinol Metab. 2006;91:3404-10.

9. Rosenberg IR. Summary comments. Am J Clin Nutr. 1989;50:1231-3.

10. Rosenberg IH. Sarcopenia: origins and clinical relevance. J Nutr. 1997; 127:990.

11. Ferrucci L, Guralnik JM, Buchner D, et al. Departures from linearity in the relationship between measures of muscular strength and physical performance of the lower extremities: the Women's Health and Aging Study. J Gerontol A Biol Sci Med Sci. 1997;52:M275-85.

12. Messier V, Rabasa-Lhoret R, Barbat-Artigas S, et al. Menopause and sarcopenia: a potential role for sex hormones. Maturitas. 2011;68(4):331-6.

13. Rolland YM, Perry HM 3rd, Patrick P, et al. Loss of appendicular muscle mass and loss of muscle strength in young postmenopausal women. J Gerontol A Biol Sci Med Sci. 2007;62(3):330-5.

14. Jubrias SA, Odderson IR, Esselman PC, et al. Decline in isokinetic force with age: muscle cross-sectional area and specific force. Pflugers Arch. 1997;434(3):246-53.

15. Cruz-Jentoft AJ, Baeyens JP, Bauer JM, et al. Sarcopenia: European consensus on definition and diagnosis: report of the European Working Group on Sarcopenia in Older People. Age Ageing. 2010;39:412-23.

16. Malmstrom TK, Morley JE. SARC-F: a simple questionnaire to rapidly diagnose sarcopenia. J Am Med Dir Assoc. 2013;14(8):531-2. 
17. Liu CJ, Latham NK. Progressive resistance strength training for improving physical function in older adults. Cochrane Database Syst Rev. 2003;3:CD002759.

18. Rossouw JE, Anderson GL, Prentice RL, et al. Risks and benefits of estrogen plus progestin in healthy postmenopausal women: principal results from the women's health initiative randomized controlled trial. JAMA. 2002;288(3):321-33.

19. Gokhale JA, Frenkel SR, Dicesare PE. Estrogen and osteoarthritis. Am J Orthop. 2004;33:71-80.

20. Von Muhlen D, Morton D, Von Muhlen CA, et al. Postmenopausal estrogen and increased risk of clinical osteoarthritis at the hip, hand, and knee in older women. J Womens Health Gend Based Med. 2002;11:511-8.

21. Reginster JY, Beaudart C, Buckinx F, et al. Osteoporosis and sarcopenia: two diseases or one? Curr Opin Clin Nutr Metab Care. 2016;19(1):31.

22. Zacker RJ. Health-related implications and management of sarcopenia. JAAPA. 2006;19(10):24-9.

23. Manini T, Clark B. Dynapenia and Aging: an Update. J Gerontol A Biol Sci Med Sci. 2012;67A(1):28-40.

24. Parr EB, Coffey VG, Hawley JA. 'Sarcobesity': a metabolic conundrum. Maturitas. 2013;74(2):109-13.

25. Fried LP, Ferrucci L, Darrer J, et al. Untangling the concepts of disability, frailty and comorbidity: implications for improved targeting and care. J Gerontol A Biol Sci Med Sci. 2004;59(3):255-63.

26. Abellan van Kan G, Rolland Y, Bergman H, et al. The I.A.N.A. Task Force on frailty assessment of older people in clinical practice. J Nutr Health Aging. 2008;12:29-37.

27. Abellan van Kan G, Rolland YM, Morley JE, et al. Frailty: toward a clinical definition. J Am Med Dir Assoc. 2008;9:71-2.

28. Khandelwal D, Goel A, Kumar U, et al. Frailty is associated with longer hospital stay and increased mortality in hospitalized older patients. J Nutr Health Aging. 2012;16(8):732-5.

29. Patil Sapna S, Hasamnis Ameya A, Jena SK, et al. Low Awareness of Osteoporosis Among Women Attending an Urban Health Centre in Mumbai, Western India. Malays J Public Health Med. 2010;10:6-13.
Publisher's Note Springer Nature remains neutral with regard to jurisdictional claims in published maps and institutional affiliations.

\section{About the Author}

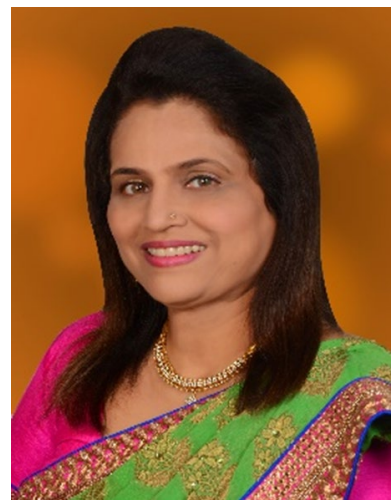

Prof. Suvarna Satish Khadilkar is the editor in chief of this journal. She is currently working as Consultant Gyne-Endocrinologist, and Professor and Head of the Department of Obstetric and Gynecology, Bombay Hospital Institute of Medical Sciences and Medical Research Centre, Mumbai. In the past, she worked as an Associate Professor and Unit Chief at JJ Group of Hospitals and Grant Medical College [GMC], Mumbai, and thereafter in the capacity of the Professor and Head in Department of

Obstetrics and Gynecology, Government Medical College, Kolhapur, Maharashtra. She is an undergraduate and postgraduate teacher and examiner, Mumbai University and Maharashtra University of Health Sciences over past 30 years. Pursuing her interest in endocrinology, she acquired Diploma in Endocrinology from the prestigious University of South Wales, UK, and has been appointed as a recognized teacher in endocrinology in University of South Wales. She has been appointed as a member of FIGO working group on post-reproductive health. She is the Treasurer of FOGSI. She has held many prestigious positions like President of Indian Menopause Society, Chairperson of Reproductive Endocrinology Committee of FOGSI, President, Association of Medical Women in India, Mumbai. She is currently the librarian of Mumbai Ob-Gyn Society. She has published six textbooks and more than 70 articles at national and international levels. She is the recipient of 30 awards for her research work including the Young Scientist Award. 